\title{
Insulin autoimmune syndrome induced by methimazole in a Korean girl with Graves' disease
}

\author{
Sun Hee Lee, MD', \\ Seung Hwan Oh, MD², \\ Woo Yeong Chung, MD ${ }^{1}$ \\ Departments of ${ }^{1}$ Pediatrics and \\ ${ }^{2}$ Laboratory Medicine, \\ Inje University Busan Paik Hospital, \\ Inje University College of Medicine, \\ Busan, Korea
}

\begin{abstract}
Hypoglycemia was detected in a 15-year-old girl due to loss of consciousness. She was diagnosed with Graves' disease and was being treated with methimazole for the past 4 months. A paradoxically increased insulin levels was found when she suffered from the hypoglycemic episode. An imaging study showed no mass lesion in the pancreas, and insulin antibodies were found in the serum. She was diagnosed with insulin autoimmune syndrome. Her HLA typing was performed, and it revealed HLA-DRB1 *04:06. The patient was treated with a corticosteroid for 2 months. After discontinuing the steroid, the insulin antibody titer decreased dramatically, and she did not have any episode of hypoglycemia since. This is the first report of insulin autoimmune syndrome in a Korean girl, and we have revealed the connection between HLA type and insulin autoimmune syndrome in Korea.
\end{abstract}

Keywords: Autoimmune syndrome, Graves' disease, Methimazole, Hypoglycemia, Adolescents

\section{Introduction}

Hypoglycemia is rare in childhood. However, delay in the diagnosis or inadequate management of hypoglycemia during childhood may be more harmful to a developing brain ${ }^{11}$. Insulin autoimmune syndrome or Hirata disease is a rare condition characterized by hyperinsulinemic hypoglycemia associated with a high titer of antibodies against endogenous insulin in the absence of pathologic abnormalities of the pancreatic islets and exposure to exogenous insulin ${ }^{2}$. The majority of cases of insulin autoimmune syndrome have been reported in $\mathrm{Japan}^{2}$. A history of other autoimmune disorders, particularly Graves' disease, and treatment with sulfhydryl medications, particularly methimazole, is common in insulin autoimmune syndrome.

In this paper, we report a 15-year-old girl with fasting hypoglycemia, hyperinsulinemia, and a high titer of anti-insulin antibodies due to drug-induced autoimmune hypoglycemia. This report is the first case of insulin autoimmune syndrome in adolescents in Korea.

\section{Case report}

A 15-year-old girl was found to have markedly decreased plasma glucose level $(30 \mathrm{mg} / \mathrm{dL})$ resulting in loss of consciousness. She was admitted to Inje University Busan Paik Hospital for further investigation and treatment. It was her first hypoglycemic episode. She was diagnosed with Graves' disease four months prior. And is being treated with methimazole and propranolol since then. Except for Graves' disease, there was no specific past medical history.

The physical exam was unremarkable, except for the patient having a grade II goiter and mild exophthalmos. Laboratory investigations initially revealed high serum glucose levels $(210 \mathrm{mg} /$ $\mathrm{dL}$ ), but fasting hypoglycemia was later detected. The fasting blood sugar was $39 \mathrm{mg} / \mathrm{dL}$ (normal range, 70 to $100 \mathrm{mg} / \mathrm{dL})$, with an insulin level of $238.2 \mu \mathrm{U} / \mathrm{mL}(2.6$ to $24.9 \mu \mathrm{U} / \mathrm{mL})$ and a

\author{
Address for correspondence: \\ oo Yeong Chung, MD \\ Inje University Busan Paik Hospital, \\ Inje University College of Medicine \\ 75 Bokji-ro, Busanjin-gu, \\ Busan 614-735, Korea \\ Tel: +82-51-890-6280 \\ Fax: +82-51-895-7785 \\ E-mail: chungwy@chol.com
}


C-peptide level of $8.46 \mathrm{ng} / \mathrm{dL}$ ( 1.1 to $4.4 \mathrm{ng} / \mathrm{mL}$ ). At the time of the hypoglycemia, her cortisol level was $16.89 \mu \mathrm{g} / \mathrm{dL}$, growth hormone level was $20.4 \mathrm{ng} / \mathrm{mL}$, and serum ketone was negative. Further laboratory studies showed a normal complete blood count, normal electrolytes, and normal renal and liver function tests. The thyroid function test was abnormal: free T4 $2.81 \mathrm{ng} /$ dL, T3 $171.2 \mathrm{ng} / \mathrm{dL}$, thyroid-stimulating hormone $<0.005 \mu \mathrm{IU} /$ $\mathrm{mL}$, thyroglobulin $\mathrm{Ab}>4,000 \mathrm{IU} / \mathrm{mL}$ (normal, $<115 \mathrm{IU} / \mathrm{mL}$ ), antimicrosomal antibody $88.72 \mathrm{IU} / \mathrm{mL}$ (normal, $<34 \mathrm{IU} / \mathrm{mL}$ ), TBII 49.1\% (normal, $<15 \%$ ), and thyroid-stimulating antibody $874.6 \%$ (negative, $<140 \%$ ).

No antiglutamic acid decarboxylase antibodies, nor anti-islet cell antibodies were detected. Hemoglobin A1c of 5.4\% were revealed. Anti-insulin antibody titer of $>100 \mathrm{U} / \mathrm{mL}$ (normal, $<5 \mathrm{U} / \mathrm{mL}$ ) was detected. Oral glucose tolerance test was performed due to the fluctuating blood glucose levels, but the result was normal.

Computed tomography of the abdomen was negative for a pancreatic tumor. Based on the episodes of hypoglycemia accompanied by extremely high levels of insulin, C-peptide, and the presence of anti-insulin antibodies to endogenous human insulin, the patient was diagnosed with insulin autoimmune syndrome. Since she has been taking methimazole; an insulin autoimmune syndrome related to methimazole was suspected. The serologic typing of HLA alleles showed that the patients was HLA-DRB1 ${ }^{\star}$ 04:06.

She was started on oral hydrocortisone $50 \mathrm{mg} / \mathrm{m}^{2}$, with slow tapering over 2 months. The patient responded well to the treatment, with resolution of the hypoglycemia and a decrease in the insulin autoantibody titers (Fig. 1). Because propylthiouracil can induce hepatotoxicity in Graves' disease, she continued to take methimazole. Even though she continued to use methimazole for her Graves' disease, her anti-insulin antibody titer decreased to $14 \mathrm{U} / \mathrm{mL}$ (normal, $<5 \mathrm{U} / \mathrm{mL}$ ) one month after stopping the hydrocortisone, and she never had

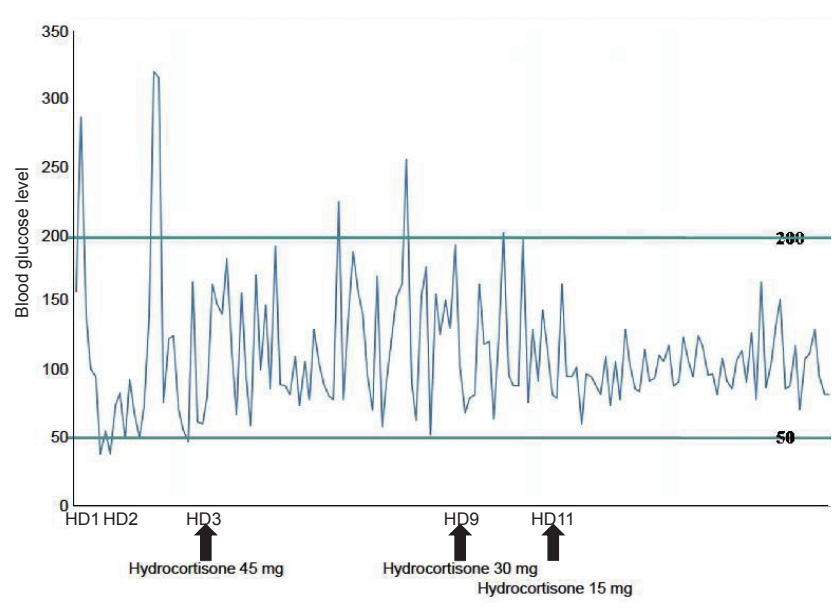

Fig. 1. Blood glucose level in the patient. There were fluctuations of blood glucose levels from hypoglycemia (39 mg/dL) to hyperglycemia (up to $321 \mathrm{mg}$ / $\mathrm{dL}$ ). After taking oral hydrocortisone, the variability of blood glucose levels was decreased. During tapered hydrocortisone, there was no hypoglycemia. hypoglycemia again.

\section{Discussion}

Insulin autoimmune syndrome was first described by Hirata in $1970^{3)}$. It is characterized by episodes of hyperinsulinemic hypoglycemia that occur most often postprandially. In Japan, insulin autoimmune syndrome is the third leading cause of severe hypoglycemia after insulinoma and extrapancreatic neoplasm ${ }^{4)}$. However, it is extremely uncommon in Western countries $^{5)}$.

Most of the patients diagnosed with insulin autoimmune syndrome have associated comorbidities or exposure to various medications. In non-Asian patients, this syndrome is often associated with rheumatologic disease, such as systemic lupus erythematosus or rheumatoid arthritis. Some insulin autoimmune syndrome patients have an underlying hematologic disease, such as benign monoclonal gammopathy or multiple myeloma ${ }^{5}$.

In approximately $47 \%$ of non-Asian patients, insulin autoimmune syndrome appears to be triggered by exposure to different medications (captopril, penicillamine, pyritinol, carbimazole, imipenem, propylthiouracil, hydralazine, procainamide, isoniazid, and penicillin $\mathrm{G}$ ), except for medications containing a sulfhydryl group. However, a clear connection between medications containing a sulfhydryl group and the occurrence of insulin autoimmune syndrome was revealed in Japanese cases $^{5)}$. Approximately $41 \%$ of Asian patients with insulin autoimmune syndrome were exposed to medications containing a sulfhydryl group. A relationship between methimazole, which contains a sulfhydryl group, for the treatment of Graves' disease and insulin autoimmune syndrome is well known ${ }^{6}$. However, other Japanese patients developed insulin autoimmune syndrome in response to tolbutamide, gold thioglucose, interferon alpha, steroids, antihypertensive drugs (captopril and diltiazem), nonsteroidal anti-inflammatory drugs (loxoprofen sodium and diclofenac sodium), and tolperisone ${ }^{3,4)}$. A case of insulin autoimmune syndrome associated with the use of alpha-lipoic acid was reported in $2007^{7}$. In this case, our patient was also diagnosed with Graves' disease and had been treated with methimazole.

Insulin autoimmune syndrome is a disorder that is strongly associated with $\mathrm{HLA}^{8)}$. An association of insulin autoimmune syndrome with HLA-DR4 was described, and most HLA-DR4positive patients with insulin autoimmune syndrome possess HLA-DRB $1^{*} 0406$, which is rare in Caucasians ${ }^{8}$. It is well known that Graves' disease patients who carry DRB $1^{\star} 0406$ developed insulin autoimmune syndrome after they took methimazole ${ }^{9)}$. The DRB1 ${ }^{\star} 0406$ allele appears to play an important role in presenting insulin peptides to $\mathrm{T}$ cells ${ }^{10)}$. Matsushita et al. ${ }^{11)}$ indicated that a reducing compound, such as methimazole, may cleave the disulfide bond in vivo and allow the DRe cleave the disulfide bond i-presenting cells to bind much of the linear fragment of the insulin A chain, which may lead to the 
activation of self insulin-specific T helper cells. Our patient also had the $\mathrm{DRB}^{\star}{ }^{\star} 0406$ allele.

Although hypoglycemic episodes are the most important and impressive phenomena of insulin autoimmune syndrome, hyperglycemia may paradoxically occur immediately following a meal or oral glucose challenge. Moreover, a few insulin autoimmune cases manifested as diabetic ketoacidosis followed by recurrent hypoglycemia ${ }^{12)}$. The hyperglycemia is caused by insulin antibodies binding to the insulin secreted in response to rising blood glucose levels after a meal. This binding reduces the availability of the secreted insulin for the receptors in the liver and peripheral tissues, resulting in hyperglycemia and further insulin secretion ${ }^{5}$. Fluctuations of blood glucose levels from hypoglycemia to hyperglycemia were also present in our patient, but her hemoglobin Alc was in the normal range, and the oral glucose tolerance test was normal.

In the majority of patients, hypoglycemia improves or resolves completely. Most patients are treated for symptomatic hypoglycemia. The first line of treatment is low-carbohydrate meals to prevent postprandial hypoglycemia. Some patients are treated with steroids, primarily oral prednisone. Other medications, such as acarbose, somatostatin, and diazoxide, have been tried, with variable results. In some cases in which hypoglycemia was induced by medication, discontinuing the suspected drug led to resolution of the symptoms ${ }^{5)}$. Our patient was treated with oral steroids, resulting in the resolution of hypoglycemia and decreased the insulin antibody titer. Despite continuing to take methimazole, the patient's insulin antibody titer was decreased, and no hypoglycemic episode appeared. There was one report similar to our case. Okabe et al. ${ }^{13)}$ reported a patient with Graves' disease and insulin autoimmune syndrome that continued the methimazole treatment. The insulin antibody titer was reduced and hypoglycemic episodes disappeared. In that report, they suggested that the disappearance of hypoglycemic symptoms may be due to the immunomodulatory effect of methimazole. However, more studies are needed regarding the continued use of methimazole in insulin autoimmune syndrome patients with Graves' disease.

In Korea, there have been several reports regarding insulin autoimmune syndrome ${ }^{14,15)}$. All cases were reported in adults. Two cases were associated with the use of methimazole for Graves' disease ${ }^{16,17)}$, one case was associated with N-acetylcysteine, which contains a sulfhydryl group ${ }^{15)}$, and one case was associated with alpha-lipoic acid ${ }^{18)}$. Only one report revealed a connection with HLA-DR typing in Korea ${ }^{18)}$.

This is the first report of insulin autoimmune syndrome related to methimazole in Korean adolescents. Our patient had the $\mathrm{DRB1}{ }^{\star} 0406$ allele, which is related to insulin autoimmune syndrome. Her hypoglycemic event was successfully treated with a steroid, while she continued to take methimazole.

\section{Conflict of interest}

No potential conflict of interest relevant to this article was reported.

\section{References}

1. Menni F, de Lonlay P, Sevin C, Touati G, Peigne C, Barbier $\mathrm{V}$, et al. Neurologic outcomes of 90 neonates and infants with persistent hyperinsulinemic hypoglycemia. Pediatrics 2001;107:476-9.

2. Basu A, Service FJ, Yu L, Heser D, Ferries LM, Eisenbarth G. Insulin autoimmunity and hypoglycemia in seven white patients. Endocr Pract 2005;11:97-103.

3. Uchigata Y, Eguchi Y, Takayama-Hasumi S, Omori Y. Insulin autoimmune syndrome (Hirata disease): clinical features and epidemiology in Japan. Diabetes Res Clin Pract 1994;22:89-94.

4. Uchigata Y, Hirata Y. Insulin autoimmune syndrome (IAS, Hirata disease). Ann Med Interne (Paris) 1999;150:245-53.

5. Lupsa BC, Chong AY, Cochran EK, Soos MA, Semple RK, Gorden P. Autoimmune forms of hypoglycemia. Medicine (Baltimore) 2009;88:141-53.

6. Hirata Y. Methimazole and insulin autoimmune syndrome with hypoglycemia. Lancet 1983;2:1037-8.

7. Uchigata Y. The novel agent, alpha lipoic acid, can cause the development of insulin autoimmune syndrome. Intern Med 2007;46:1321-2.

8. Uchigata Y, Kuwata S, Tokunaga K, Eguchi Y, TakayamaHasumi S, Miyamoto M, et al. Strong association of insulin autoimmune syndrome with HLA-DR4. Lancet 1992;339:393-4.

9. Uchigata Y, Kuwata S, Tsushima T, Tokunaga K, Miyamoto M, Tsuchikawa K, et al. Patients with Graves' disease who developed insulin autoimmune syndrome (Hirata disease) possess HLA-Bw62/Cw4/DR4 carrying DRB1 ${ }^{\star} 0406$. J Clin Endocrinol Metab 1993;77:249-54.

10. Virally ML, Timsit J, Chanson P, Warnet A, Guillausseau PJ. Insulin autoimmune syndrome: a rare cause of hypoglycaemia not to be overlooked. Diabetes Metab 1999;25:429-31.

11. Matsushita S, Takahashi K, Motoki M, Komoriya K, Ikagawa S, Nishimura Y. Allele specificity of structural requirement for peptides bound to $\mathrm{HLA}-\mathrm{DRB} 1^{\star} 0405$ and $-\mathrm{DRB} 1^{\star} 0406$ complexes: implication for the HLAassociated susceptibility to methimazole-induced insulin autoimmune syndrome. J Exp Med 1994;180:873-83.

12. Jo YG, Kim YI, Lee SJ, Kim KW, Chun SW, Kim YJ, et al. Insulin Autoimmune Syndrome with Diabetic Ketoacidosis. J Korean Diabetes 2012;13:105-9.

13. Okabe R, Inaba M, Hosoi M, Ishimura E, Kumeda Y, Nishizawa $Y$, et al. Remission of insulin autoimmune syndrome in a patient with Grave's disease by treatment with methimazole. Intern Med 1999;38:482-5.

14. Cho BY, Lee HK, Koh CS, Min HK. Spontaneous hypoglycemia and insulin autoantibodies in a patient with Graves' disease. Diabetes Res Clin Pract 1987;3:119-24.

15. Kim DJ, Kim NH, Seo JA, Kim SG, Choi KM, Baik SH, et al. A case of autoimmune insulin syndrome. Korean J Med 2006;71:683-7. 
16. Lee KS, Kim JH, Choi WH, Kim TW, Kim MH. A case report of insulin autoimmune syndrome in graves' disease. J Korean Soc Endocrinol 1993;8:451-5.

17. Lim JK, Woo YA, Kang SJ, Yoo SS, Hong KY, Kim SH. Ensulin Autoimmune Syndrome in a Patient with
Methimazole-Treated Graves' Disease: A Case report. J Korean Soc Endocrinol 1998;13:612-6.

18. Chang HJ, Choi HS, Park MY, Leem SM, Jang YS, Park KS, et al. A case of insulin autoimmune syndrome related to alpha-lipoic acid. Korean J Med 2009;76:600-4. 